\title{
The expressions of carbonic anhydrase 9 and vascular endothelial growth factor in astrocytic tumors predict a poor prognosis
}

\author{
HEON YOO ${ }^{1 *}$, SEIL SOHN ${ }^{1 *}$, BYUNG HO NAM ${ }^{2}$, HYE SOOK MIN ${ }^{3}$, EUGENE JUNG ${ }^{1}$, \\ SANG HOON SHIN ${ }^{1}$, HO-SHIN GWAK ${ }^{1}$ and SEUNG HOON LEE ${ }^{1}$ \\ ${ }^{1}$ Neuro-Oncology Clinic, Center for Specific Organs Cancer; ${ }^{2}$ Cancer Registration and Biostatistics Branch; \\ ${ }^{3}$ Department of Pathology, National Cancer Center, Goyang, Republic of Korea
}

Received December 31, 2009; Accepted February 17, 2010

DOI: 10.3892/ijmm_00000427

\begin{abstract}
Hypoxia in the tumor microenvironment triggers a variety of genetic and adoptive responses that regulate tumor growth. Tumor hypoxia is often associated with a malignant phenotype, resistance to therapy, and poor survival. The objectives of this study were to evaluate the expressions of carbonic anhydrase 9 (CA9) and vascular endothelial growth factor (VEGF) in astrocytic gliomas and to relate patterns of expression with prognosis, that is with histological grade and survival. We investigated 78 World Health Organization (WHO) grade II, III, and IV astrocytic gliomas. CA9 expression was examined in paraffin-embedded sections by immunohistochemistry. Fourteen tumors were grade II, 30 were grade III, and 34 were grade IV. It was found that CA9 expression was significantly associated with a higher-grade histology $(\mathrm{p}<0.001)$. There were 3 CA9 positive tumors in grade II (21.4\%), 10 in grade III (33.3\%), and 27 in grade IV (79.4\%). For all tumors and WHO grade II, overall survival was found to be significantly dependent on CA9 expression ( $\mathrm{p}=0.004$, $\mathrm{p}=0.01$ ). Furthermore, VEGF expression was found to be significantly related to tumor grade $(\mathrm{p}=0.02)$ and tended to be related to overall survival $(\mathrm{p}=0.1)$. However, no relation was found between the expression of CA9 and VEGF $(p=0.17)$. Nevertheless, the expressions of CA9 and VEGF were found to be associated with tumor grade and possibly with survival. Further studies on a larger patient population are needed to
\end{abstract}

Correspondence to: Dr Seung Hoon Lee, Neuro-Oncology Clinic, National Cancer Center, 809 Madu-1 dong, Ilsandong-gu, Goyang, Gyonggi 411-769, Republic of Korea

E-mail: nslsh@ncc.re.kr

"Contributed equally

Abbreviations: CA, carbonic anhydrase; VEGF, vascular endothelial growth factor; WHO, World Health Organization; FGF, fibroblast growth factor; DNET, dysembryoplastic neuroepithelial tumor; HIF, Hypoxia inducible factor

Key words: carbonic anhydrase 9, hypoxia, glioma, vascular endothelial growth factor determine the correlation between the expressions of CA9, and VEGF in astrocytic gliomas and clinical outcome.

\section{Introduction}

Current treatment options for astrocytic gliomas, are limited to surgical resection, radiation therapy and chemotherapy, and these modalities are frequently inadequate. A better understanding of the molecular mechanisms and microenvironmental factors that drive glioma tumor growth are required to promote developments of more effective therapies for glioma patients. One major factor that contributes to enhanced glycolysis is adaptation to hypoxia in the tumor microenvironment (1). Hypoxia in tumors triggers molecular responses that promote an aggressive cancer phenotype, and intratumoral hypoxia has been associated with a poor clinical outcome for many cancers (2-4). Furthermore, hypoxic tumor cells also contribute to resistance, radiation and chemotherapy $(5,6)$, and hypoxia regulates several important tumor growth-promoting cellular mechanisms, such as angiogenesis, cellular proliferation, genomic instability, and tumor invasion (7).

Carbonic anhydrase 9 (CA9) is induced by hypoxia in a wide range of tumors, and it is usually suppressed under normoxic conditions (8-11). Expressed CA9 colocalizes with pimonidazole, a chemical marker of hypoxia, and its expression is usually restricted to perinecrotic areas $(8,12)$, which make CA9 an attractive endogenous marker of tumor hypoxia. Furthermore, CA9 expression has been associated with a poor prognosis in lung, breast, and cervical cancer (13-15), and with resistance to chemotherapy in head and neck cancers (16). Previously, we reported that CA9 is associated with anaplastic phenotypes in meningioma and suggested that it is an indicator of a poor prognosis in affected patients (17). However, the association between CA9 and astrocytic glial tumor grade, and its prognostic value in human astrocytic gliomas have not been well investigated.

The formation of primitive blood vessels from progenitors, such as hemangioblasts and angioblasts, is dependent on vascular endothelial growth factor (VEGF) and the VEGF receptor system (18). Malignant glioma cells secrete a variety of angiogenic factors such as VEGF and basic fibroblast growth factor (FGF) (19). Furthermore, VEGF has been considered to play a major role in the angiogenesis, as suggested of other solid tumors such as colon and breast 


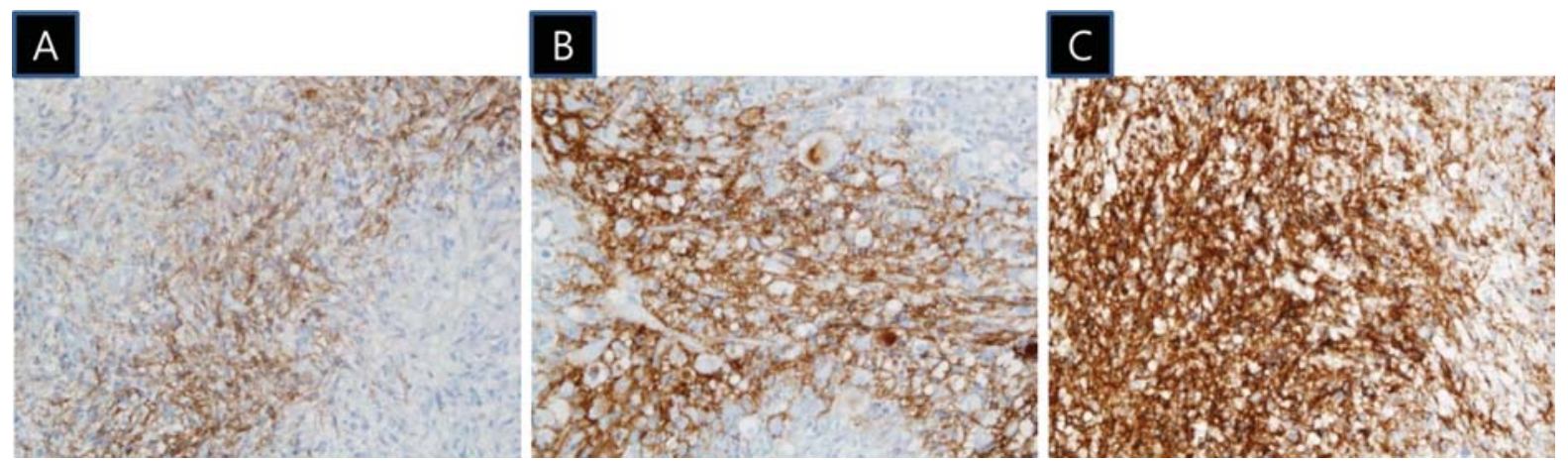

Figure 1. Immunoexpression of CA9. CA9 was weakly stained in the cytoplasm of tumor cells (A, intensity, 1) and moderately (B, intensity, 2). In some cases a strong positivity (C, intensity, 3) was observed adjacent to geographic necrosis. (x200, A-C).
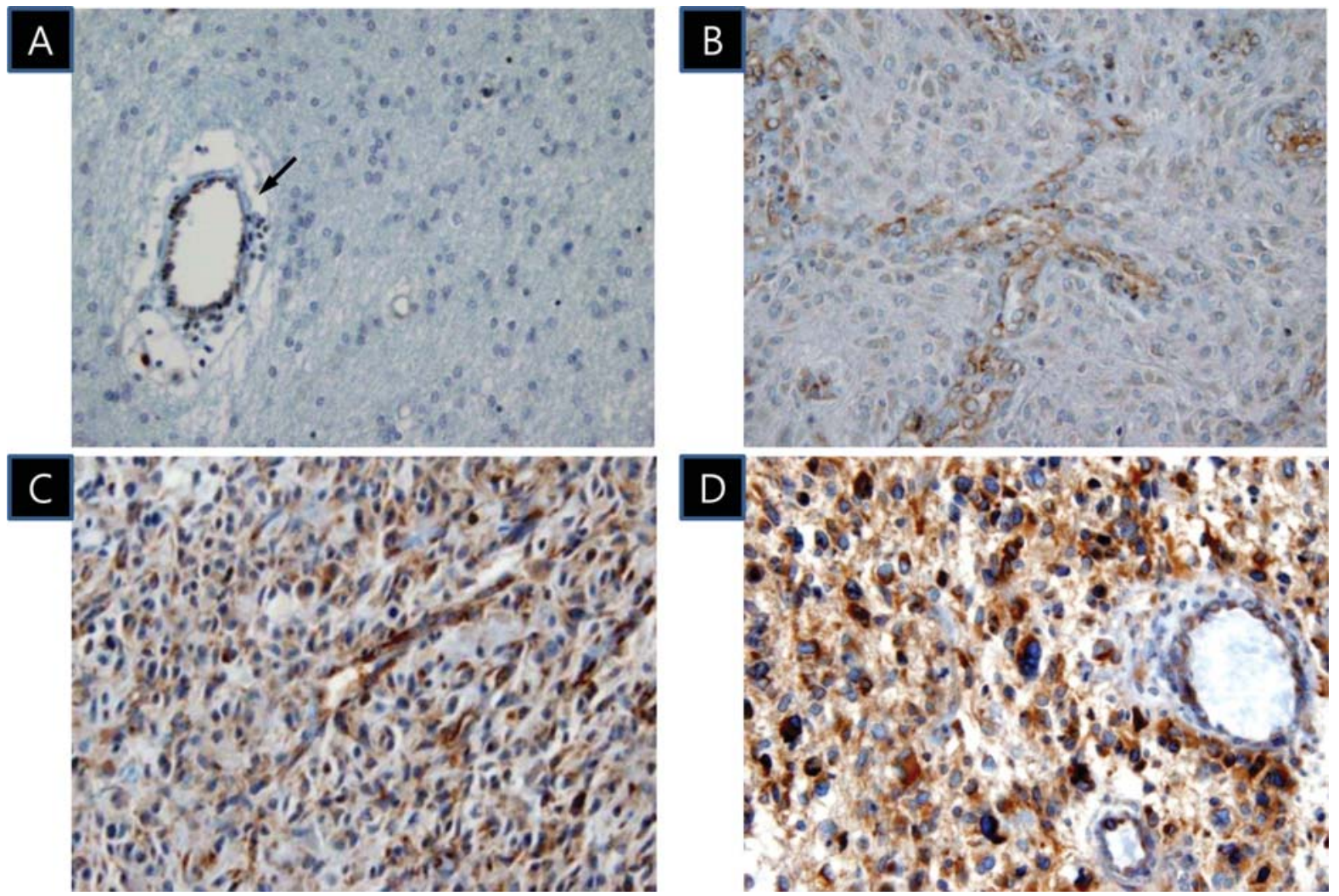

Figure 2. Immunoexpression of VEGF. Only endothelial cells were positive in (A, arrow, score, 0 ) as a positive control for VEGF immunohistochemistry. As shown (B-D), VEGF was weakly expressed (B, score, 1), moderately (C, score, 2), or showed marked positivity compared to blood vessels (D, score, 3) (x400, A-D).

carcinomas (20), and VEGF expression has been shown to influence the outcomes of other tumors (21-23). However, the relation between VEGF and WHO tumor grade and the effect of VEGF expression on prognosis has not been investigated in depth for astrocytic gliomas.

\section{Materials and methods}

Patients. We analyzed 78 pathologically confirmed astrocytic gliomas with archived tissues available for immunohistochemistry staining. Of the 78 tumors 14 were WHO grade II, 30 were grade III, and 34 were grade IV. Of the 78 patients 42 were male, and 56 patients were $<60$ years old. CA9 was positive in 40 patients, and negative in 38 . VEGF was positive in 33 patients and negative in 43 (Table I).

Tumor samples and immunohistochemistry. Slides stained with hematoxylin and eosin $(\mathrm{H} \& \mathrm{E})$ retained tissues were reviewed by a neuropathologist (H.S. Min). Immunohistochemical staining was performed automatically using Benchmark XT (Roche, Switzerland) which utilized the conventional streptavidin-biotinperoxidase method, as recommended by the manufacturer. CA9 (Novus, Polyclonal, 1:1,000) and VEGF (BD Pharmingen, G153-694, 1:500) primary antibodies were used. Staining intensities of CA9 positive cells in tumor parenchyma were assessed using an arbitrary scoring system (Fig. 1) and CA9 
A

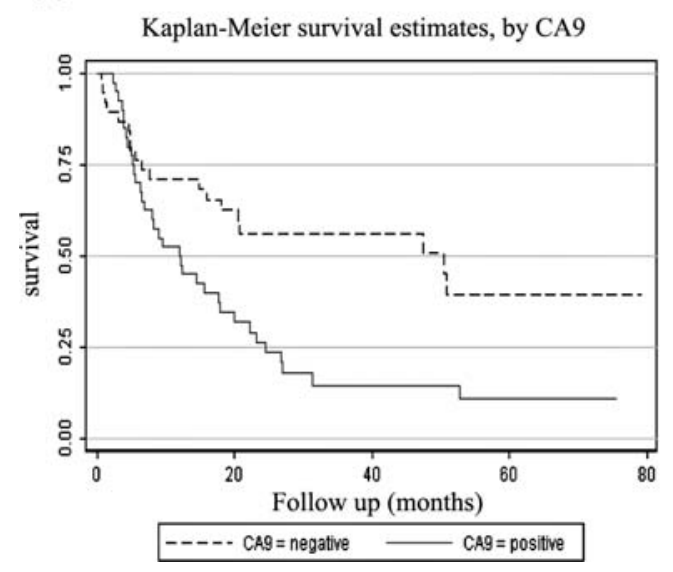

B

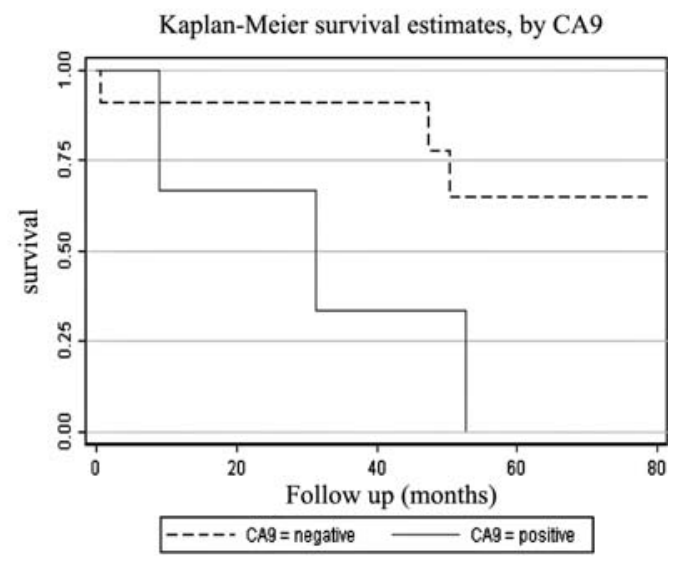

Figure 3. Survival by CA9. (A) Survival by CA9 in all WHO grades (p=0.004). (B) Survival by CA9 in WHO grade II (p=0.03).

Table I. Clinical characteristics.

\begin{tabular}{lc}
\hline Characteristics & No. of patients (\%) \\
\hline Age & \\
$<60$ & $56(71.8)$ \\
$\geq 60$ & $22(28.2)$ \\
Gender & \\
Male & $42(53.8$ \\
Female & $36(46.2)$ \\
CA9 & \\
Negative & $38(48.7)$ \\
Positive & $40(51.3)$ \\
VEGF & \\
Negative & $43(56.6)$ \\
Positive & $33(43.4)$ \\
Grade & \\
II & $14(17.9)$ \\
III & $30(38.5)$ \\
IV & $34(43.6)$ \\
\hline
\end{tabular}

staining scores were calculated by multiplying positive cell percentage scores $(1,<10 \% ; 2,10 \%-50 \% ; 3,>50 \%)$ by intensity scores $(0$, null; 1 , weak; 2 , moderate; 3 , strong). CA9 expression was considered negative when CA9 staining scores were 0 or 1 , and positive when scores were $>1$. For VEGF, positive immunoexpression was graded as $+1,+2$, or +3 based on staining intensity (Fig. 2). VEGF scores of 0 or 1 were considered negative for VEGF expression and scores of 2 or 3 were considered positive.

Statistical analysis. Pearson's correlation coefficients were used to examine correlations between CA9 and VEGF scores and tumor grades.

For survival analysis, overall survival was defined as time from date of surgery until death, which was considered an event in the analysis. Univariate and multivariate Cox- regression analysis, Kaplan-Meier survival analysis, the logrank test, Wilcoxon's (Breslow's) test, and Spearman's correlation were used.

Statistical analysis was performed using Stata 10 software (Stata Corporation Station, TX, USA). Statistical significance was accepted for a p-value of 0.05 .

Ethics. The study protocol was approved by the Institutional Review Board of the National Cancer Center, Korea. (Protocol number NCCNCS-09-227).

\section{Results}

Univariate Cox-regression survival analysis. Survival analysis was performed on age, gender, CA9, VEGF, WHO grade. The parameters found to adversely affect survival time were age, CA9 positivity, and WHO grade (Table IIA).

Multivariate Cox-regression survival analysis. We considered that age, CA9, VEGF, and WHO grade potentially affected survival time based on the results of univariate Cox-regression analysis. Multivariate Cox-regression analysis was performed for CA9, and VEGF separately, and both analyses showed that histologic grade was the only factor that significantly affected survival (Table IIB and C).

Relation between CA9 positivity and WHO tumor grade. CA9 expression was analyzed in the 78 astrocytic gliomas. Mean intensities of CA9 scores for different WHO grades are shown in Table IIIA. CA9 was positive in 3 patients of WHO grade II (21.4\%), 10 of grade III (33.3\%), and 27 of grade IV (79.4\%), and the correlation between the prevalence of CA9 positivity and WHO grade was significant (Pearson's correlation coefficient $=19.6, \mathrm{p}<0.001)$.

Relation between VEGF positivity and WHO grade. VEGF expression was analyzed in 76 of the 78 gliomas. Positivity of VEGF by WHO grade is presented in Table IIIB. VEGF expression was positive in 2 patients with WHO grade II $(14.3 \%), 12$ in grade III (42.9\%), and 19 in grade IV (55.9\%), and this correlation between the prevalence of VEGF positivity 
Table II. Survival analysis.

A, Univariate Cox-regression survival analysis

\begin{tabular}{lccc}
\hline & P-value & Hazard ratio & 95\% CI for hazard ratio \\
\hline Age & 0.036 & 1.891 & $1.042-3.430$ \\
Gender & 0.565 & 1.173 & $0.681-2.020$ \\
CA9 & 0.005 & 2.267 & $1.285-4.000$ \\
VEGF & 0.109 & 1.565 & $0.906-2.704$ \\
WHO Grade III & 0.109 & 2.158 & $0.843-5.524$ \\
WHO Grade IV & $<0.001$ & 6.610 & $2.559-17.073$ \\
\hline
\end{tabular}

B, Multivariate Cox-regression survival analysis

\begin{tabular}{lccc}
\hline Characteristics & P-value & Hazard ratio & 95\% CI for hazard ratio \\
\hline Age & 0.557 & 1.216 & $0.634-2.331$ \\
CA9 & 0.554 & 1.227 & $0.623-2.417$ \\
WHO Grade III & 0.129 & 2.088 & $0.808-5.401$ \\
WHO Grade IV & 0.003 & 5.369 & $1.794-16.069$ \\
\hline
\end{tabular}

C, Multivariate Cox-regression survival analysis

\begin{tabular}{lccc}
\hline Characteristics & P-value & Hazard ratio & 95\% CI for hazard ratio \\
\hline Age & 0.725 & 1.126 & $0.582-2.178$ \\
VEGF & 0.945 & 1.021 & $0.574-1.815$ \\
WHO Grade III & 0.104 & 2.209 & $0.851-5.734$ \\
WHO Grade IV & $<0.001$ & 6.195 & $2.252-17.044$ \\
\hline
\end{tabular}

and WHO grade was also significant (Pearson's correlation coefficient $=7.0, \mathrm{p}=0.03$ ). However, CA9 and VEGF positivity did not correlate with each other $(\mathrm{p}=0.17)$.

Survival according to CA9 expressions. Survival analysis was performed by Kaplan-Meier curve analysis using the CA9 scores of all 78 tumors. Median survival time was 50.4 months for CA9 negative patients, but 12.0 months for CA9-positive patients, and these survival times were significantly different $(\mathrm{p}=0.004)$ (Fig. 3A).

Survival analysis was also performed for WHO grade II, III, and IV tumors separately. Median survival time was not reached for CA9-negative grade II patients, and 31 months for CA9-positive grade II patients. Similarly, median survival times for grade III patients, were 50.8 and 22.3 months for CA9-negative and -positive patients, and median survival times for grade IV patients were 4.8 and 9.6 months for CA9negative and -positive patients.

For grade II patients, survival times were significantly different for CA9-positive and -negative patients $(\mathrm{p}=0.01)$ (Fig. 3B). However, this was not the case for grade III, or IV patients who were not significantly different between CA9positive and -negative group in WHO grade III and IV patients (grade III, $\mathrm{p}=0.6$; grade IV, $\mathrm{p}=0.5$ ).

Survival according to VEGF expression. Survival analysis was performed using the Kaplan-Meier method according to VEGF grade for all 78 tumors. Median survival times were 26.8 and 14.4 months in VEGF-negative and -positive patients, respectively. However, this difference was not significant $(\mathrm{p}=0.1)($ Fig. 4).

\section{Discussion}

Currently, the importance of the role played by hypoxia in the biology of tumors is evident. Research efforts continue to identify intrinsic markers of hypoxia and both CA9 and VEGF are known to be products of hypoxia-induced pathways. CA9 is a transmembrane protein, and converts carbon dioxide to bicarbonate and hydrogen, and thus regulates microenvironmental $\mathrm{pH}$, and influences other processes such as cell to cell adhesion, cellular proliferation, and tumor cell invasion (24). Correlations between clinical outcomes and CA9 expression have been reported for several malignancies such as for cervix (25), lung (13), breast (26), kidney (27) and head and neck (28). Furthermore, CA9 expression has been associated with poorer relapse-free and overall survival in invasive breast carcinoma (14), and in small cell lung cancer, CA9-positive tumors have been reported to be associated with a significantly shorter overall survival (13). Previously, we suggested that CA9 is a prognostic factor in meningioma (17). Thus, CA9 expression clearly has prognostic significance in a variety of human tumors.

In the present study, the CA9 positive rate among patients with an astrocytic glioma was found to increase significantly 


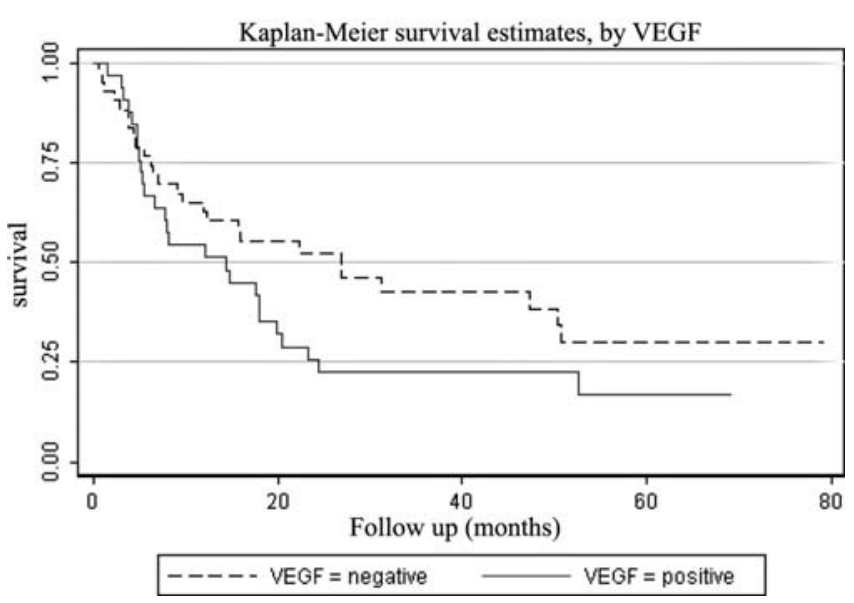

Figure 4. Survival by VEGF in all WHO grade tumors. Survival of VEGFnegative group was better than that of VEGF-positive group $(\mathrm{p}=0.1)$.

Table III. Correlation between CA9, VEGF and WHO grade.

A, Correlation between CA9 and WHO grade

\begin{tabular}{|c|c|c|c|c|}
\hline & \multicolumn{3}{|c|}{ Grade } & \multirow[t]{2}{*}{ Total } \\
\hline & II & III & IV & \\
\hline \multicolumn{5}{|l|}{ Negative } \\
\hline No. & 11 & 20 & 7 & 38 \\
\hline$\%$ of total grade & $78.6 \%$ & $66.7 \%$ & $20.6 \%$ & $48.7 \%$ \\
\hline \multicolumn{5}{|l|}{ Positive } \\
\hline No. & 3 & 10 & 27 & 40 \\
\hline$\%$ of total grade & $21.4 \%$ & $33.3 \%$ & $79.4 \%$ & $51.3 \%$ \\
\hline \multicolumn{5}{|l|}{ Total } \\
\hline No. & 14 & 30 & 34 & 78 \\
\hline$\%$ of total grade & $100 \%$ & $100 \%$ & $100 \%$ & $100 \%$ \\
\hline
\end{tabular}

Pearson correlation coefficient $=19.6, \mathrm{p}<0.001$.

B, Correlation between VEGF and WHO grade

\begin{tabular}{lcccc}
\hline & \multicolumn{3}{c}{ Grade } & Total \\
\cline { 2 - 3 } & II & III & IV & \\
\hline Negative & & & & \\
No. & 12 & 16 & 15 & 43 \\
$\%$ of total grade & $85.7 \%$ & $57.1 \%$ & $44.1 \%$ & $56.6 \%$ \\
$\begin{array}{l}\text { Positive } \\
\text { No. }\end{array}$ & 2 & 12 & 19 & 33 \\
$\%$ of total grade & $14.3 \%$ & $42.9 \%$ & $55.9 \%$ & $43.4 \%$ \\
$\begin{array}{l}\text { Total } \\
\text { No. }\end{array}$ & 14 & 28 & 34 & 76 \\
$\%$ of total grade & $100 \%$ & $100 \%$ & $100 \%$ & $100 \%$ \\
\hline
\end{tabular}

Pearson correlation coefficient $=7.0, \mathrm{p}=0.03$. as WHO grade increased. Furthermore, CA9 positivity was found to be associated with poor survival for all WHO grades. In the present study, CA9 was found to be expressed in perinecrotic tumoral regions which suggests that CA9 expression is induced by hypoxic conditions in human brain astrocytic glioma. Degree of necrosis increases with WHO grade and typically perinecrotic regions within tumors are hypoxic. However, CA9 expression may not precisely mimic oxygen concentrations as measured by microelectrodes or by bioreductive drugs. In the present study, our separate analysis of WHO grade II, III, and IV tumors, showed that survival times were significantly different according to CA9 status for WHO grade II tumors, but not for WHO grade III, and IV tumors. Furthermore, the survival time of CA9-negative tumors was significantly greater than that of CA9-positive tumors, which is possibly because survival times or patients with a WHO grade III and IV tumor were primarily influenced by factors other than CA9 status.

Relations between CA9 expressional status and hypoxia or pH may explain the correlation between CA9 expressional status and tumor grade. CA9 expression is the response to hypoxia mediated by HIF-1 transcription factor and its levels in tumor tissues have been reported to provide a measure of chronic hypoxia (8). Furthermore, tumor cell proliferation involves the consumption of oxygen and leads to increased acid production (29), and CA9 upregulation under hypoxic conditions eliminates this acid load and endows tumor cells with a survival advantage which contributes to the acquisition of a more aggressive phenotype (30). These findings suggest that CA9 is a marker of tumor aggressiveness. In the present study, univariate analysis indicated that CA9 is a prognostic factor of survival, but multivariate analysis identified WHO grade as the only significant prognostic factor. These findings suggest that CA9 expression is a separate indicator of brain tumor survival, which does not reflect the same features as WHO grade, and that thus, perhaps CA9 expression should be considered as another classification criterion for astrocytic glioma.

Malignant glioma is a highly vascularized tumor and its microvascular density has been reported to be correlated with a poor clinical prognosis. Furthermore, malignant glioma cells secrete a variety of angiogenic factors such as VEGF and basic FGF (19). Furthermore, VEGF has been considered to play a major role in angiogenesis and is a prognostic factor for other solid tumors such as those of colon cancer and breast carcinoma (22,31). In the present study, VEGF expression was found to be significantly correlated with WHO tumor grade, but this significance was weaker than that found for CA9 expression. These findings are similar to those of a previous report in which VEGF expression was found to be significantly higher in glioblastoma than in low grade glioma, however the strength of these relations were weaker than those of CA9 expression (32). During our survival analysis, the survival of patients with a VEGF-negative tumor was found to be non-significantly greater than that of those with a VEGFpositive tumor $(\mathrm{p}=0.1)$. Furthermore, VEGF expression was not found to be a significant factor of survival time by univariate and multivariate regression analysis, which indicates that a larger scale study is needed to determine the effect of VEGF on survival. In addition, our findings concur with a 
previous report that the prognostic significance of VEGF expression did not withstand multivariate analysis (30).

At least four mechanisms have been suggested for the upregulation of VEGF gene expression in glioma. One involves a hypoxia/HIF-related mechanism in growing glioma tissues and another a HuR protein-related mechanism. According to this mechanism, VEGF is related with hypoxia and CA9. The other two mechanisms involve the EGFR signaling pathway and FoxM1B transcription factor. The EGFR signaling pathway stimulates VEGF gene expression via a HIF-independent mechanism, and it has been reported that FoxM1B is upregulated in glioblastoma multiform tissues, but not in lowgrade astrocytoma tissues, and that it stimulates VEGF expression independently of HIF (33). Furthermore, it has been argued that different degrees of hypoxia are required for induction of VEGF and CA9 and that CA9 induction requires more severe hypoxia. Thus, the presence of CA9 expression reflects exposure to prolonged hypoxia (13). These findings help explain why VEGF expression was not found to be correlated with CA9 expression and is less significantly related to WHO grade than CA9.

Likewise, in the present study, we found that VEGF was not an independent prognostic factor of survival. Bevacizumab in combination with cytotoxic agents such as irinotecan, and other anti-angiogenic drugs such as VEGF-Trap has recently been reported to suppress tumor growth and improve survival in malignant glioma patients during phase II clinical trials $(34,35)$. These drugs require further investigation to prove their abilities to enhance survival, and in addition, more study of drugs targeting CA9 is warranted.

In summary, we found that intratumoral CA9 expression is associated with WHO grade in astrocytic gliomas, and that it is possibly an independent prognostic factor of overall survival. Furthermore, VEGF expression was found to be correlated with WHO grade, but not found to be a prognostic factor of overall survival. Our findings suggest that therapeutic strategies for the treatment of glioma involving the targeting of CA9 are required and that therapied targeting VEGF require more investigation. In addition, VEGF expression was not found to be correlated with CA9 expression, which suggests that VEGF is produced independently of CA9. Finally, we suggest that further study be undertaken to determine the correlation between CA9 and clinical outcome in patients with astrocytic glioma of the brain.

\section{Acknowledgements}

This work was supported by the National Cancer Center Grant 0410052-3.

\section{References}

1. Robin ED, Murphy BJ and Theodore J: Coordinate regulation of glycolysis by hypoxia in mammalian cells. J Cell Physiol 118: 287-290, 1984.

2. Haapasalo JA, Nordfors KM, Hilvo M, et al: Expression of carbonic anhydrase IX in astrocytic tumors predicts poor prognosis. Clin Cancer Res 12: 473-477, 2006.

3. Hockel M, Schlenger K, Aral B, Mitze M, Schaffer U and Vaupel P: Association between tumor hypoxia and malignant progression in advanced cancer of the uterine cervix. Cancer Res 56: 4509-4515, 1996.

4. Brizel DM, Sibley GS, Prosnitz LR, Scher RL and Dewhirst MW: Tumor hypoxia adversely affects the prognosis of carcinoma of the head and neck. Int J Radiat Oncol Biol Phys 38: 285-289, 1997.
5. Nordsmark M, Loncaster J, Aquino-Parsons C, et al: Measurements of hypoxia using pimonidazole and polarographic oxygen-sensitive electrodes in human cervix carcinomas. Radiother Oncol 67: 35-44, 2003.

6. Fyles AW, Milosevic M, Wong R, et al: Oxygenation predicts radiation response and survival in patients with cervix cancer. Radiother Oncol 48: 149-156, 1998.

7. Harris AL: Hypoxia - a key regulatory factor in tumour growth. Nat Rev Cancer 2: 38-47, 2002.

8. Lal A, Peters H, St Croix B, et al: Transcriptional response to hypoxia in human tumors. J Natl Cancer Inst 93: 1337-1343, 2001.

9. Olive PL, Aquino-Parsons C, MacPhail SH, et al: Carbonic anhydrase 9 as an endogenous marker for hypoxic cells in cervical cancer. Cancer Res 61: 8924-8929, 2001.

10. Beasley NJ, Wykoff CC, Watson PH, et al: Carbonic anhydrase IX, an endogenous hypoxia marker, expression in head and neck squamous cell carcinoma and its relationship to hypoxia, necrosis, and microvessel density. Cancer Res 61: 5262-5267, 2001.

11. Wykoff CC, Beasley N, Watson PH, et al: Expression of the hypoxia-inducible and tumor-associated carbonic anhydrases in ductal carcinoma in situ of the breast. Am J Pathol 158: 1011-1019, 2001.

12. Airley RE, Loncaster J, Raleigh JA, et al: GLUT-1 and CAIX as intrinsic markers of hypoxia in carcinoma of the cervix: relationship to pimonidazole binding. Int J Cancer 104: 85-91, 2003.

13. Giatromanolaki A, Koukourakis MI, Sivridis E, et al: Expression of hypoxia-inducible carbonic anhydrase- 9 relates to angiogenic pathways and independently to poor outcome in non-small cell lung cancer. Cancer Res 61: 7992-7998, 2001.

14. Chia SK, Wykoff CC, Watson PH, et al: Prognostic significance of a novel hypoxia-regulated marker, carbonic anhydrase IX, in invasive breast carcinoma. J Clin Oncol 19: 3660-3668, 2001.

15. Loncaster JA, Harris AL, Davidson SE, et al: Carbonic anhydrase (CA IX) expression, a potential new intrinsic marker of hypoxia: correlations with tumor oxygen measurements and prognosis in locally advanced carcinoma of the cervix. Cancer Res 61: 6394-6399, 2001

16. Koukourakis MI, Giatromanolaki A, Sivridis E, et al: Hypoxiaregulated carbonic anhydrase-9 (CA9) relates to poor vascularization and resistance of squamous cell head and neck cancer to chemoradiotherapy. Clin Cancer Res 7: 3399-3403, 2001.

17. Yoo H, Baia GS, Smith JS, et al: Expression of the hypoxia marker carbonic anhydrase 9 is associated with anaplastic phenotypes in meningiomas. Clin Cancer Res 13: 68-75, 2007.

18. Ferrara N, Gerber HP and LeCouter J: The biology of VEGF and its receptors. Nat Med 9: 669-676, 2003.

19. Saleh M, Stacker SA and Wilks AF: Inhibition of growth of C6 glioma cells in vivo by expression of antisense vascular endothelial growth factor sequence. Cancer Res 56: 393-401, 1996.

20. Shibuya M: Brain angiogenesis in developmental and pathological processes: therapeutic aspects of vascular endothelial growth factor. FEBS J 276: 4636-4643, 2009.

21. Jacobsen J, Grankvist K, Rasmuson T, Bergh A, Landberg G and Ljungberg B: Expression of vascular endothelial growth factor protein in human renal cell carcinoma. BJU Int 93: 297-302, 2004

22. Linderholm BK, Lindh B, Beckman L, et al: Prognostic correlation of basic fibroblast growth factor and vascular endothelial growth factor in 1307 primary breast cancers. Clin Breast Cancer 4: 340-347, 2003.

23. Uehara M, Sano K, Ikeda H, et al: Expression of vascular endothelial growth factor and prognosis of oral squamous cell carcinoma. Oral Oncol 40: 321-325, 2004.

24. Winum JY, Rami M, Scozzafava A, Montero JL and Supuran C: Carbonic anhydrase IX: a new druggable target for the design of antitumor agents. Med Res Rev 28: 445-463, 2008.

25. Lee S, Shin HJ, Han IO, et al: Tumor carbonic anhydrase 9 expression is associated with the presence of lymph node metastases in uterine cervical cancer. Cancer Sci 98: 329-333, 2007.

26. Brennan DJ, Jirstrom K, Kronblad A, et al: CA IX is an independent prognostic marker in premenopausal breast cancer patients with one to three positive lymph nodes and a putative marker of radiation resistance. Clin Cancer Res 12: 6421-6431, 2006.

27. Atkins M, Regan M, McDermott D, et al: Carbonic anhydrase IX expression predicts outcome of interleukin 2 therapy for renal cancer. Clin Cancer Res 11: 3714-3721, 2005. 
28. Choi SW, Kim JY, Park JY, Cha IH, Kim J and Lee S: Expression of carbonic anhydrase IX is associated with postoperative recurrence and poor prognosis in surgically treated oral squamous cell carcinoma. Hum Pathol 39: 1317-1322, 2008.

29. Ivanov SV, Kuzmin I, Wei MH, et al: Down-regulation of transmembrane carbonic anhydrases in renal cell carcinoma cell lines by wild-type von Hippel-Lindau transgenes. Proc Natl Acad Sci USA 95: 12596-12601, 1998.

30. Korkolopoulou P, Perdiki M, Thymara I, et al: Expression of hypoxia-related tissue factors in astrocytic gliomas. A multivariate survival study with emphasis upon carbonic anhydrase IX. Hum Pathol 38: 629-638, 2007

31. Preusser M, Wolfsberger S, Haberler C, et al: Vascularization and expression of hypoxia-related tissue factors in intracranial ependymoma and their impact on patient survival. Acta Neuropathol 109: 211-216, 2005.
32. Said HM, Hagemann C, Staab A, et al: Expression patterns of the hypoxia-related genes osteopontin, CA9, erythropoietin, VEGF and HIF-1alpha in human glioma in vitro and in vivo. Radiother Oncol 83: 398-405, 2007.

33. Zhang Y, Zhang N, Dai B, et al: FoxM1B transcriptionally regulates vascular endothelial growth factor expression and promotes the angiogenesis and growth of glioma cells. Cancer Res 68: 8733-8742, 2008.

34. Vredenburgh JJ, Desjardins A, Herndon JE II, et al: Phase II trial of bevacizumab and irinotecan in recurrent malignant glioma. Clin Cancer Res 13: 1253-1259, 2007.

35. Zuniga RM, Torcuator R, Jain R, et al: Efficacy, safety and patterns of response and recurrence in patients with recurrent high-grade gliomas treated with bevacizumab plus irinotecan. J Neurooncol 91: 329-336, 2009. 